\title{
Editorial
}

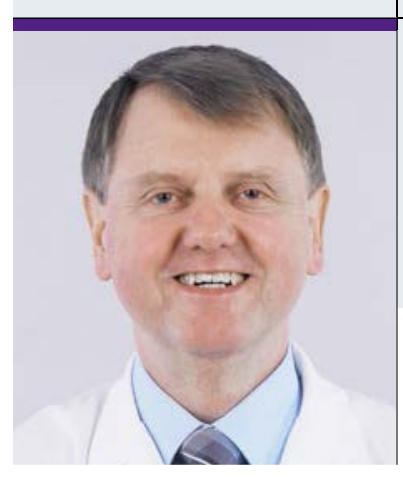

"Grundlage für die ethische Bewertung der Pränatalmedizin

sollte nicht die Methode selbst, sondern der ärztliche Umgang

mit dieser Methode sein."

Prof. Dr. med. Josef Wisser

Facharzt für Gynäkologie und Geburtshilfe, Klinik für Geburtshilfe, UniversitätsSpital Zürich

\section{Biografie der fetalen Therapie}

$\mathrm{D}$ er Einsatz der Ultraschalltechnologie zur Darstellung des Feten durch Ian Donald 1958 hat die Tür zur Entwicklung der vorgeburtlichen Medizin eröffnet. Über ein Jahrzehnt standen den diagnostischen Bemühungen keine therapeutischen Möglichkeiten zur Seite, sodass der pränatalen Diagnostik einschließlich der zytogenetischen Abklärung des Feten, welche seit 1966 durch Amniozentese möglich war, der Makel der „Abtreibungsmedizin" anhaftete. Pränataldiagnostik wurde als „Teufelswerk" gebrandmarkt, was jedoch völlig verkennt, dass nicht die Methode, sondern der ärztliche Umgang mit dieser Methode, Grundlage für die ethische Bewertung ist.

\section{Der ungeborene Patient}

Glücklicherweise hat sich bereits in den 1980er-Jahren das Konzept des „Feten als Patient" mehr und mehr durchgesetzt. Erste sehr erfolgreiche Behandlungen des Feten haben Eingang in den klinischen Alltag gefunden, wie beispielsweise die fetale Lungenreifung mittels Steroidgabe an die Mutter bei drohender Frühgeburtlichkeit und die intrauterine Bluttransfusion zunächst intraperitoneal, dann intravasal zur Behandlung der fetalen Anämie bei Blutgruppenunverträglichkeit oder fetaler Parvovirusinfektion. Die Ausdehnung der diagnostischen Möglichkeiten auf detaillierte morphologische und funktionelle Befunde hat das Spektrum der therapeutischen Optionen erweitert. Es wurden minimalinvasive Behandlungsstrategien entwickelt und für spezifische Erkrankungsbilder wie das fetofetale Transfusionssyndrom monochorialer diamnioter Gemini hat sich die fetoskopisch durchgeführte Laserkoagulation der plazentaren Gefäße seit fast zwei Jahrzehnten fest etabliert.

Das Studium der perinatalen Physiologie der Wehentätigkeit und deren pränataler Kontrolle hat Operationen am offenen Uterus möglich gemacht, die derzeit zunehmend klinische Verbreitung in der Behandlung der fetalen Spina bifida finden. Die vielen Versuche einer Therapie genetischer Erkrankungen des Feten sind bislang über einen experimentellen Charakter nicht hinausgekommen. Erste erfolgreiche Ansätze beispielsweise in der Behandlung einer fetalen Osteogenesis imperfecta sind kürzlich publiziert worden, sodass auch hier eine weitere Entwicklung zu erwarten ist.

Für den Pränatalmediziner ist der Fetus zweifelsfrei ein ungeborener Patient. Durch die Behandlung von Mutter und Fetus ist der vorgeburtlich tätige Arzt gezwungen, sich sehr genau über die Konsequenzen seines Handelns für die beiden Patienten Rechenschaft abzulegen. So gesehen ist die vorgeburtliche Medizin ein Paradebeispiel reflektierender, patientenzentrierter Medizin geworden.

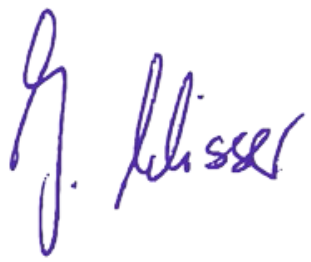

